\title{
Approche variationnelle de l'endommagement : I. Les concepts fondamentaux
}

\author{
Kim Pham ${ }^{\text {a }}$ Jean-Jacques Marigo ${ }^{\mathrm{b}}$ \\ ${ }^{a}$ Institut Jean le Rond d'Alembert (UMR 7190), Université Paris VI \\ ${ }^{\mathrm{b}}$ Laboratoire de Mécanique des Solides (UMR 7649), École Polytechnique
}

\section{Résumé}

On s'intéresse aux modèles d'endommagement de type fragile où la variable d'endommagement scalaire est régie par une loi à seuil. Considérant ici des modèles locaux, on rappelle que le postulat de Drucker-Ilyushin impose que le modèle soit nécessairement standard, ce qui fournit automatiquement un cadre variationnel à l'écriture du problème d'évolution. On peut alors définir naturellement les concepts de durcissement, d'adoucissement et de stabilité. On aboutit ainsi à une formulation basée sur les trois principes physiques d'irréversibilité, de stabilité et de bilan d'énergie, directement généralisable à des modèles non locaux et se prêtant à un traitement mathématique rigoureux.

Abstract. The variational approach to damage : I. The foundations. This Note is devoted to the construction of local brittle damage laws. We first justify by the Drucker-Ilyushin postulate to set their formulation within the framework of Generalized Standard Materials. Accordingly, the strain work becomes a state function whose convexity properties are directly related to the hardening or softening properties of the material. Moreover, the evolution problem can be read as a variational problem which is reinforced so that it finally contains the concepts of stability and of energy conservation.

Key words: Damage, stability, variational methods

Mots-clés : Endommagement, stabilité, méthodes variationnelles

\section{Abridged English version}

We first introduce the main assumptions entering in the definition of a brittle damage model:

(i) The damage state of the material point is characterized by a scalar internal variable $\alpha$ growing from 0 to $\alpha_{m} \leq+\infty, 0$ corresponding to the undamaged state and $\alpha_{m}$ to the full damaged state;

(ii) At a given $\alpha$, the behavior is elastic and defined by the elastic potential $\psi(\varepsilon, \alpha)$, positively homogeneous of degree 2 and strictly convex function of the strain state $\varepsilon$, see (1). The dual potential giving the strain state in terms of the stress state $\sigma$ is denoted by $\psi^{*}(\sigma, \alpha)$ and is a positively homogeneous of degree 2 and strictly convex function of $\sigma$, see (2). Moreover $\psi$ is continuously differentiable and its partial derivative with respect to $\alpha$ is negative, see (3);

Email addresses: pham@lmm.jussieu.fr (Kim Pham), marigo@lms.polytechnique.fr (corresponding author) (Jean-Jacques Marigo). 
(iii) For irreversibility reason $\alpha$ can only increase and its rate-independent evolution is governed by the yield function $\phi(\varepsilon, \alpha)$ and the Kuhn-Tucker conditions

$$
\dot{\alpha} \geq 0, \quad \phi(\varepsilon, \alpha) \leq 0, \quad \dot{\alpha} \phi(\varepsilon, \alpha)=0 .
$$

If we adopt the Drucker-Ilyushin postulate requiring that the strain work is non negative in any strain cycle, then it can be proved [6] that the yield function is necessarily given by

$$
\phi(\varepsilon, \alpha)=-\frac{\partial \psi}{\partial \alpha}(\varepsilon, \alpha)-\kappa(\alpha)
$$

where $\kappa(\alpha)>0$. That means that the criterion of damage evolution is governed by the principle of critical elastic energy release rate. The fundamental consequence is that the strain work is the state function given by $W(\varepsilon, \alpha)=\psi(\varepsilon, \alpha)+d(\alpha)$, where $d(\alpha)=\int_{0}^{\alpha} \kappa(\beta) d \beta$ represents the energy dissipated by the material point when its damage state grows from 0 to $\alpha$. Accordingly, the stress-strain relation and the evolution damage law can read as (9) which is the cornerstone of the variational formulation of the damage evolution in a body. Moreover, the elastic domains $\mathcal{R}(\alpha)$ and $\mathcal{R}^{*}(\alpha)$ in the strain and stress spaces are directly expressed in terms of $W$ and its Legendre transform $W^{*}$, see (11). We can then define the different concepts of hardening or softening in terms of the growth or the decrease of $\mathcal{R}(\alpha)$ and $\mathcal{R}^{*}(\alpha)$, see Definition 2.2. It turns out that these hardening properties can be expressed in terms of convexity properties of the state functions $W$ and $W^{*}$ provided that one chooses the dissipated energy $d$ as the damage variable, see Proposition 2.3.

Let $\Omega$ be a body submitted to a $t$-dependent loading. Let $\mathcal{C}_{t}=U_{t}+\mathcal{C}^{0}$ be the affine space of admissible displacement fields at time $t$, where $\mathcal{C}^{0}$ is a linear space and $U_{t}$ is a given displacement field. Let $\mathcal{D}_{0}=$ $\left\{\alpha: 0 \leq \alpha \leq \alpha_{m}\right.$ in $\left.\Omega\right\}$ be the convex set of admissible damage fields. The damage fields which are accessible from the damage field $\alpha$ are restricted by the irreversibility condition and are the elements of $\mathcal{D}(\alpha)=\left\{\beta: \alpha \leq \beta \leq \alpha_{m}\right.$ in $\left.\Omega\right\}$. The energy of the body at time $t$ in the admissible state $(v, \beta)$ is defined by (15) where $W_{t}^{e}$ denotes the potential of the applied forces. By virtue of the standard character of the model, the evolution problem is equivalent to find, for all $t \geq 0$, the displacement and damage fields $\left(u_{t}, D_{t}\right)$ which satisfy the three items (ir), (st) and (eb). However, in the case of softening materials, it turns out that the evolution problem can have an infinite number of solutions or solutions which are not smooth in time (even if the model is regularized by non local terms, see [2]). Therefore, we propose to reinforce the item (st), which can be considered as a first order stability condition, by the item (ST) which corresponds to the concept of directional stability, see Definition 3.3. However, let us note that the energy of the current state is only compared to the energy of accessible states because of the irreversibility condition. Since (eb) has no meaning when the evolution is discontinuous in time, we propose to replace it by (EB) which is equivalent to (eb) when the evolution is smooth and which still has a sense when the evolution is discontinuous. It is an energy balance condition which requires that the total energy of the body be continuous even if the state undergoes a jump discontinuity. In essence the three items (IR), (ST) and (EB) which constitute the final version of the formulation of the evolution problem are identical to those which are used in the variational approach to fracture [3] or more generally in the modern mathematical treatment of rate independent laws [7]. This new formulation will be the basis of the qualitative analysis of non local softening laws, purpose of our future publications.

\section{Introduction}

Nous revisitons la théorie phénoménologique de l'endommagement introduite par Kachanov en l'étudiant sous l'angle des concepts mathématiques modernes attachés aux approches variationnelles. Il s'avère que toutes les lois de comportement de type standards généralisés et indépendantes des vitesses (rate independent) se prêtent à ce formalisme qui, en retour, permet d'en faire une analyse qualitative rationnelle [7], [3]. Nous l'utilisons ici pour construire des lois d'endommagement fragile non régularisées mais l'objectif est évidemment de l'étendre aux lois non locales [4], ce qui fera l'objet de la deuxième partie de cette étude. Le caractère standard de ces lois fait que le problème d'évolution quasi-statique 
s'écrit naturellement sous forme variationnelle, dans la lignée des travaux de Nguyen Q. S. [8] et de façon plus générale de l'École française créée par Germain. L'apport des développements récents [7] [3] des approches variationnelles est de faire ressortir trois principes physiques fondamentaux en formulant le problème d'évolution quasi-statique sous la forme de trois items : (i) une condition d'irréversibilité de l'évolution, (ii) une condition de stabilité des états, (iii) un bilan d'énergie. Ainsi, les concepts de stabilité font partie intégrante de la formulation. De plus, le bilan d'énergie est écrit différemment de façon à pouvoir l'utiliser même pour des évolutions irrégulières en temps. On a ainsi une formulation qui se prête à des traitements mathématiques et numériques rigoureux. Tout cela s'avérera décisif pour l'étude des lois d'endommagement adoucissantes (même régularisées) où les cas de multiplicité et de non régularité de solutions sont plus la règle que l'exception [2].

\section{Les modèles d'endommagement fragile non régularisés}

2.1. Le cadre général. Suivant la démarche initiée par [5], la construction d'un modèle d'endommagement passe par le choix : (i) d'un paramètre d'endommagement ; (ii) de la dépendance de la relation contraintedéformation vis-à-vis de ce paramètre; (iii) de la loi d'évolution du paramètre. L'objectif de l'étude n'étant pas de développer un modèle précis, applicable à un matériau précis, mais plutôt de fournir un cadre général, nous prenons le parti de faire les choix les plus simples tout en dégageant les propriétés génériques. Nous supposons que :

(i) l'état d'endommagement peut être décrit à l'aide d'un scalaire $\alpha$, hypothèse évidemment très simplificatrice mais qui se justifie par l'usage en calcul de structures auquel on destine ce type de modèles. Le choix du paramètre lui-même reste en grande partie arbitraire, on peut toujours en changer moyennant un changement de variable sans pour autant changer de modèle. Cette latitude sera utilisée par la suite. $\grave{A}$ ce stade, nous supposons seulement que $\alpha$ varie entre 0 et $\alpha_{m}$ avec $0<\alpha_{m} \leq+\infty, 0$ correspondant à l'état sain et $\alpha_{m}$ à l'état "ultime" d'endommagement;

(ii) à $\alpha$ fixé, le matériau a un comportement élastique. Son élasticité, dépendante de $\alpha$, est caractérisée par le potentiel élastique $\psi(\varepsilon, \alpha)$ où $\psi$ est continûment différentiable dans $\mathbb{M}_{s}^{n} \times\left[0, \alpha_{m}\right), \mathbb{M}_{s}^{n}$ désignant l'ensemble des matrices $n \times n$ symétriques, $n$ étant la dimension de l'espace. Pour rendre compte de comportement différent en traction et en compression, différence qui correspond à des fermetures de micro-fissures à une échelle plus fine, on suppose que $\psi$ n'est pas quadratique en $\varepsilon$ mais seulement positivement homogène de degré 2 , i.e.

$$
\sigma=\frac{\partial \psi}{\partial \varepsilon}(\varepsilon, \alpha), \quad \psi(k \varepsilon, \alpha)=k^{2} \psi(\varepsilon, \alpha), \quad \forall k \geq 0, \quad \forall(\varepsilon, \alpha) \in \mathbb{M}_{s}^{n} \times\left[0, \alpha_{m}\right],
$$

$\sigma$ et $\varepsilon$ représentant l'état local des contraintes et des déformations.

Cette propriété d'homogénéité peut se démontrer dans le cadre de la théorie de l'homogénéisation pour un endommagement d'un matériau linéairement élastique par microfissuration en supposant que le contact entre les lèvres des fissures est de type unilatéral et s'effectue sans frottement. Elle est perdue si le glissement entre les lèvres se fait avec frottement, on quitte alors le cadre élastique fragile proprement dit, il faut ajouter des variables internes traduisant les déformations résiduelles induites par le frottement. Ces aspects sortent du cadre de notre étude.

Nous supposons de plus que $\psi$ est une fonction strictement convexe de $\varepsilon$ à $\alpha$ fixé, $\alpha \neq \alpha_{m}$, ce qui rend la relation $\varepsilon \mapsto \sigma$ strictement monotone et donc inversible. La relation inverse $\sigma \mapsto \varepsilon$ s'obtient à partir du potentiel dual $\psi^{*}$, lui-même fonction strictement convexe de $\sigma$ et positivement homogène de degré 2 en $\sigma$ :

$$
\varepsilon=\frac{\partial \psi^{*}}{\partial \sigma}(\sigma, \alpha), \quad \psi^{*}(\sigma, \alpha)=\max _{\varepsilon^{\prime} \in \mathbb{M}_{s}^{n}}\left\{\sigma \cdot \varepsilon^{\prime}-\psi\left(\varepsilon^{\prime}, \alpha\right)\right\} .
$$

On traduit que le matériau s'assouplit quand l'endommagement augmente en demandant que

$$
\frac{\partial \psi}{\partial \alpha}(\varepsilon, \alpha) \leq 0, \quad \forall \alpha \in\left[0, \alpha_{m}\right) \text { et } \forall \varepsilon \in \mathbb{M}_{s}^{n} .
$$


Lorsque $\alpha$ atteint la valeur "ultime" $\alpha_{m}$, la perte de rigidité peut être partielle ou totale. Cela se traduit par

$$
\text { Rupture totale: } \forall \varepsilon, \psi\left(\varepsilon, \alpha_{m}\right)=0, \quad \text { Rupture partielle : } \exists \varepsilon \neq 0, \psi\left(\varepsilon, \alpha_{m}\right)=0 \text {, }
$$

mais on peut aussi considérer des cas où le matériau conserve toujours une réserve d'élasticité;

(iii) pour des raisons d'irréversibilité, l'endommagement ne peut que croître et son évolution est régie par une loi à seuil. Ceci se formalise en introduisant la fonction seuil d'endommagement $\phi(\varepsilon, \alpha)$, exprimée en termes des déformations pour pouvoir rendre compte des phénomènes d'adoucissement, et en écrivant les conditions classiques de Kuhn et Tucker :

$$
\dot{\alpha} \geq 0, \quad \phi(\varepsilon, \alpha) \leq 0, \quad \dot{\alpha} \phi(\varepsilon, \alpha)=0 .
$$

La première condition traduit l'irréversibilité, la deuxième est le critère d'endommagement, la troisième dite condition de cohérence exige que l'endommagement n'évolue que quand les déformations sont au seuil. On notera que $\alpha$ est l'unique variable interne du modèle et qu'elle sert aussi de variable d'écrouissage. La fonction $\phi$ est supposée définie et suffisamment régulière de façon à ce que $\phi(\varepsilon, \alpha) \leq 0$ définisse, pour tout $\alpha \in\left[0, \alpha_{m}\right)$, dans $\mathbb{M}_{s}^{n}$ un ensemble connexe fermé dont l'état non déformé est un point intérieur $(\phi(0, \alpha)<0)$, à frontière régulière évoluant continûment avec $\alpha$. Cet ensemble, noté $\mathcal{R}(\alpha)$, représente le domaine de réversibilité (dans l'espace des déformations) du matériau dans l'état d'endommagement $\alpha$. On peut lui associer, grâce à la relation déformation-contrainte (2), le domaine de réversibilité $\mathcal{R}^{*}(\alpha)$ dans l'espace des contraintes.

2.2. Les lois standards. A priori, les fonctions $\psi$ et $\phi$ peuvent être choisies indépendamment l'une de l'autre. Les lois standards [8] consistent à définir la loi d'endommagement, i.e. $\phi$, à partir de la force thermodynamique $-\partial \psi / \partial \alpha$ associée à $\alpha$ qui s'interprète comme un taux de restitution d'énergie élastique. Il s'avère que ceci n'a pas besoin d'être supposé, mais peut se déduire du postulat de Drucker-Ilyushin. Ce remarquable résultat, qui est le pilier de toute l'approche variationnelle développée dans cette Note, est établi de façon détaillée dans [6] ; aussi se contentera-t-on ici d'en rappeler l'énoncé. Soient $\alpha_{0}$ l'état initial d'endommagement et $t \mapsto \varepsilon(t)$ un cycle de déformation compatible imposé au point matériel, i.e. un chemin dans $\mathbb{M}_{s}^{n}$ paramétré par $t \in[0,1]$ tel que $\varepsilon(0)=\varepsilon(1)$ et $\varepsilon(t) \in \mathcal{R}(\alpha(t)) \forall t \in[0,1], t \mapsto \alpha(t)$ désignant l'évolution de l'endommagement régie par (5) durant ce cycle en déformation. Le travail de déformation durant ce cycle est donné par

$$
W=\int_{0}^{1} \frac{\partial \psi}{\partial \varepsilon}(\varepsilon(t), \alpha(t)) \cdot \dot{\varepsilon}(t) d t .
$$

Le postulat de Drucker-Ilyushin consiste à demander que $W \geq 0$ et ce quels que soient l'état initial d'endommagement et le cycle de déformation compatible envisagés. On obtient alors la

Proposition 2.1 (Lois standards et postulat de Drucker-Ilyushin) Le travail de déformation est non négatif quel que soit l'état d'endommagement initial et dans tout cycle en déformation compatible seulement si le critère d'endommagement est un critère portant sur le taux de restitution d'énergie élastique, i.e. seulement s'il existe $\kappa(\alpha)>0$ tel que $\phi(\varepsilon, \alpha)$ puisse s'écrire

$$
\phi(\varepsilon, \alpha)=-\frac{\partial \psi}{\partial \alpha}(\varepsilon, \alpha)-\kappa(\alpha) .
$$

Notons que : (i) on obtient un résultat moins fort si la variable d'endommagement n'est pas scalaire, cf [6, Théorèmes 6.5 et 6.6$]$; (ii) on peut montrer la réciproque moyennant des hypothèses sur l'écrouissage.

La première conséquence est que le travail de déformation est une fonction d'état, i.e. le travail fourni pour faire passer le matériau de l'état naturel sain $(0,0)$ à l'état $(\varepsilon, \alpha)$ est indépendant du trajet de déformation suivi. Il s'écrit :

$$
W(\varepsilon, \alpha)=\psi(\varepsilon, \alpha)+d(\alpha)
$$

où $\alpha \mapsto d(\alpha)$, primitive de $\alpha \mapsto \kappa(\alpha)$ nulle en 0 , s'interprète comme l'énergie dissipée pour endommager le matériau jusqu'au niveau $\alpha$. (Comme $d^{\prime}=\kappa>0$, l'énergie dissipée est fonction croissante de $\alpha$, le deuxième principe de la thermodynamique est automatiquement vérifié.) Ce faisant la relation contraintedéformation et la loi d'évolution peuvent s'écrire (tant que $\alpha<\alpha_{m}$ ) :

$$
\sigma=\frac{\partial W}{\partial \varepsilon}(\varepsilon, \alpha), \quad \text { et } \quad \dot{\alpha} \geq 0, \quad \frac{\partial W}{\partial \alpha}(\varepsilon, \alpha) \geq 0, \quad \dot{\alpha} \frac{\partial W}{\partial \alpha}(\varepsilon, \alpha)=0,
$$


remarque qui s'avère décisive pour la formulation variationnelle du problème d'évolution et l'établissement des propriétés énergétiques des solutions.

Remarque 1 Moyennant le changement de variable $\alpha \mapsto d=d(\alpha)$, ce qui revient à prendre pour variable d'endommagement l'énergie dissipée, le travail de déformation et le critère peuvent s'écrire

$$
\tilde{W}(\varepsilon, d)=\tilde{\psi}(\varepsilon, d)+d, \quad-\frac{\partial \tilde{\psi}}{\partial d}(\varepsilon, d) \leq 1 .
$$

2.3. Les propriétés d'écrouissage. Dans le modèle standard (7), les domaines de réversibilité dans l'espace des déformations et l'espace des contraintes s'écrivent, pour $\alpha \neq \alpha_{m}$ :

$$
\mathcal{R}(\alpha)=\left\{\varepsilon \in \mathbb{M}_{s}^{n}: \frac{\partial W}{\partial \alpha}(\varepsilon, \alpha) \geq 0\right\}, \quad \mathcal{R}^{*}(\alpha)=\left\{\sigma \in \mathbb{M}_{s}^{n}: \frac{\partial W^{*}}{\partial \alpha}(\sigma, \alpha) \leq 0\right\}
$$

où $W^{*}(\sigma, \alpha)=\psi^{*}(\sigma, \alpha)-d(\alpha)$ est la transformée de Legendre de $W(\epsilon, \alpha)$ par rapport à $\varepsilon$. Ce sont des domaines étoilés par rapport à 0 en vertu des propriétés d'homogénéité de $\psi$ et $\psi^{*}$. Ils sont bornés dans toutes les directions de $\mathbb{M}_{s}^{n}$ dans la mesure où $\frac{\partial \psi}{\partial \alpha}(\varepsilon, \alpha)<0, \forall \varepsilon \neq 0$. Mais, plus que leur forme, c'est l'évolution de leur taille avec $\alpha$ qui joue un rôle essentiel dans les propriétés qualitatives du problème d'évolution de l'endommagement dans une structure. Introduisons tout d'abord la

Définition 2.2 (Types d'écrouissage) On dit qu'il y a écrouissage en déformation si $\alpha \mapsto \mathcal{R}(\alpha)$ est croissant, qu'il y a durcissement si $\alpha \mapsto \mathcal{R}^{*}(\alpha)$ est croissant, qu'il y a adoucissement si $\alpha \mapsto \mathcal{R}^{*}(\alpha)$ est décroissant.

Ces monotonies, prises au sens large, doivent être comprises au sens de l'inclusion. Ainsi, l'écrouissage en déformation s'écrit $\alpha^{\prime}>\alpha \Rightarrow \mathcal{R}\left(\alpha^{\prime}\right) \supset \mathcal{R}(\alpha)$. Moyennant le choix de l'énergie dissipée comme variable d'endommagement (cf Remarque 1), il s'avère que ces propriétés de croissance ou de décroissance des domaines d'élasticité sont équivalentes à des propriétés de convexité ou de concavité de $\tilde{W}$ ou $\tilde{W}^{*}$ en vertu de la proposition suivante

Proposition 2.3 (Convexité et écrouissage) La condition d'écrouissage en déformation est équivalente à la stricte convexité de $\tilde{W}$ par rapport à $d$ à $\varepsilon$ fixé. La condition de durcissement est équivalente à la stricte convexité de $\tilde{W}$ par rapport au couple $(\varepsilon, d)$ (sachant que l'on suppose que $\tilde{W}$ est strictement convexe par rapport à $\varepsilon$ à d fixé). La condition d'adoucissement est équivalente à la stricte convexité de $\tilde{W}^{*}$ par rapport à $d$ à $\sigma$ fixé.

Notons toutefois que ces propriétés de convexité ne sont pas invariantes par changement de variable et qu'il faut faire le bon choix de la variable d'endommagement, alors que les propriétés d'écrouissage, elles, sont intrinsèques.

2.4. Des exemples.

Exemple 2.1. En 1D, $n=1$, le modèle le plus général que l'on puisse envisager correspond à

$$
W(\varepsilon, \alpha)=\frac{1}{2} E_{+}(\alpha)\left(\varepsilon^{+}\right)^{2}+\frac{1}{2} E_{-}(\alpha)\left(\varepsilon^{-}\right)^{2}+d(\alpha)
$$

où $\varepsilon^{ \pm}=\max \{0, \pm \varepsilon\} . E_{+}(\alpha)$ et $E_{-}(\alpha)$ représentent les modules d'Young en traction et en compression. Ils doivent décroître (au sens large) quand $\alpha$ croît. Si l'on veut que le matériau s'endommage en traction mais pas en compression, on prendra $E_{-}(\alpha)=E_{0}>0$ et $E_{+}$décroîtra de $E_{0}$ à 0 quand $\alpha$ croîtra de 0 à $\alpha_{m}$. Dans ce cas, $\mathcal{R}(\alpha)$ et $\mathcal{R}^{*}(\alpha)$ sont des intervalles non bornés du côté des compressions.

Exemple 2.2. En élasticité anti-plane, $n=2$, la question de contact unilatéral ne se posant pas, pour un matériau isotrope le modèle s'écrira

$$
W(\varepsilon, \alpha)=\mu(\alpha)\left(\varepsilon_{13}^{2}+\varepsilon_{23}^{2}\right)+d(\alpha)
$$

où la direction 3 correspond à la direction anti-plane. $\mu(\alpha)$ représente le module de cisaillement qui décroît de $\mu_{0}$ à 0 quand $\alpha$ croît de 0 à $\alpha_{m} \cdot \mathcal{R}(\alpha)$ et $\mathcal{R}^{*}(\alpha)$ sont des disques des plans $\left(\varepsilon_{13}, \varepsilon_{23}\right)$ et $\left(\sigma_{13}, \sigma_{23}\right)$, centrés à l'origine.

Exemple 2.3. En 3D, $n=3$, un modèle particulièrement simple permettant de rendre compte de comportement asymétrique consiste à poser [1]

$$
W(\varepsilon, \alpha)=\frac{1}{2} K(\alpha)\left(\operatorname{Tr} \varepsilon^{+}\right)^{2}+\frac{1}{2} K_{0}\left(\operatorname{Tr} \varepsilon^{-}\right)^{2}+\mu(\alpha) \varepsilon^{D} \cdot \varepsilon^{D}+d(\alpha)
$$


où $K(\alpha)$ et $\mu(\alpha)$ représentent le module de compressibilité et le module de cisaillement du matériau endommagé, $K_{0}$ est le module de compressibilité du matériau sain, $\varepsilon^{D}$ est le déviateur de $\varepsilon$, $\operatorname{Tr} \varepsilon$ sa trace, $\operatorname{Tr} \varepsilon^{ \pm}=\max \{0, \pm \operatorname{Tr} \varepsilon\} \cdot \mathcal{R}(\alpha)$ et $\mathcal{R}^{*}(\alpha)$ sont non bornés dans la direction des compressions hydrostatiques.

Dans tous ces exemples, on peut rendre compte de n'importe quel type d'écrouissage en jouant sur les fonctions de $\alpha$. De plus, en choisissant convenablement les relations endommagement-élasticité dans (12) ou (14), on peut rendre compte de fortes asymétries en traction et compression.

\section{L'évolution de l'endommagement dans une structure}

On considère une structure dont la configuration de référence naturelle est l'ouvert $\Omega$ de $\mathbb{R}^{n}$, qui est constituée d'un (plusieurs) matériau(x) endommageable(s) du type précédent (dans le cas hétérogène, le potentiel $W$, l'état ultime d'endommagement $\alpha_{m}$ et toutes les grandeurs afférentes dépendent de $x$ ) et qui est soumise à un chargement dépendant d'un paramètre $t \geq 0$. Le problème d'évolution quasi-statique consiste à chercher, à chaque "instant" $t$, les champs des déplacements $u_{t}$, d'endommagement $\alpha_{t}$ et des contraintes $\sigma_{t}$ vérifiant les équations d'équilibre, les conditions aux limites, les conditions initiales et la loi d'évolution de l'endommagement.

3.1. Le problème d'évolution et le problème incrémental. Essentiellement grâce au caractère standard de la loi d'évolution (9), le problème d'évolution se ramène à des inéquations variationnelles. Cela passe par quelques définitions préalables.

Définition 3.1 (Admissibilité des champs) On note $\mathcal{C}_{t}$ l'ensemble des déplacements cinématiquement admissibles à l'instant $t, \mathcal{C}_{t}$ est supposé affine, de la forme $U_{t}+\mathcal{C}^{0}$ où $U_{t}$ est un champ donné dépendant régulièrement de $t$ et $\mathcal{C}^{0}$ est un espace vectoriel (indépendant de $t$ ). On note $\mathcal{D}_{0}=\{\alpha: 0 \leq \alpha \leq$ $\alpha_{m}$ dans $\left.\Omega\right\}$ l'ensemble des champs d'endommagement admissibles. $\grave{A} \alpha \in \mathcal{D}_{0}$ on associe l'ensemble $\mathcal{D}(\alpha)$ des champs d'endommagement accessibles depuis $\alpha$, restreints par la condition d'irréversibilité : $\mathcal{D}(\alpha)=\left\{\beta: \alpha \leq \beta \leq \alpha_{m}\right.$ dans $\left.\Omega\right\}$.

À un couple $(v, \beta) \in \mathcal{C}_{t} \times \mathcal{D}_{0}$ admissible à l'instant $t$ on associe l'énergie totale $\mathcal{P}_{t}(v, \beta)$ de la structure dans cet état

$$
\mathcal{P}_{t}(v, \beta)=\int_{\Omega} W(x, \varepsilon(v)(x), \beta(x)) d x-W_{t}^{e}(v)
$$

où $\varepsilon(v)$ désigne la partie symétrique du gradient de $v$ et $W_{t}^{e}$ est le potentiel des efforts extérieurs donnés à

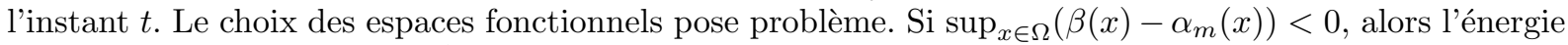
est finie dès lors que $v \in H^{1}\left(\Omega, \mathbb{R}^{n}\right)$. Par contre, les questions de régularité spatiales deviennent plus délicates dès qu'une partie de la structure est totalement endommagée. Nous ne les aborderons pas ici et raisonnerons formellement.

Se donnant le champ d'endommagement initial $\alpha_{0}$, on commence par déterminer $u_{0}$ en résolvant le problème d'élasticité linéaire associé, i.e. $u_{0}=\operatorname{argmin}_{v \in \mathcal{C}_{0}} \mathcal{P}_{0}\left(v, \alpha_{0}\right)$ et on vérifie qu'il est compatible avec le critère d'endommagement, i.e. $\varepsilon\left(u_{0}\right)(x) \in \mathcal{R}\left(x, \alpha_{0}(x)\right)$ pour tout $x \in \Omega$. Puis, si cette compatibilité est satisfaite, le problème d'évolution consiste à trouver, pour tout $t>0,\left(u_{t}, \alpha_{t}\right) \in \mathcal{C}_{t} \times \mathcal{D}_{0}$ vérifiant les 3 items suivants

$$
\begin{aligned}
\text { (ir) }: \dot{\alpha}_{t} \geq 0 \\
\text { (st) }: \mathcal{P}_{t}^{\prime}\left(u_{t}, \alpha_{t}\right)\left(v-u_{t}, \beta-\alpha_{t}\right) \geq 0, \quad \forall(v, \beta) \in \mathcal{C}_{t} \times \mathcal{D}\left(\alpha_{t}\right), \\
\text { (eb) }: \mathcal{P}_{t}^{\prime}\left(u_{t}, \alpha_{t}\right)\left(0, \dot{\alpha}_{t}\right)=0
\end{aligned}
$$

où $\mathcal{P}_{t}^{\prime}\left(u_{t}, \alpha_{t}\right)(v, \beta):=\left.\frac{d}{d h} \mathcal{P}_{t}\left(u_{t}+h v, \alpha_{t}+h \beta\right)\right|_{h=0}$ désigne la dérivée directionnelle de $\mathcal{P}_{t}$ en $\left(u_{t}, \alpha_{t}\right)$ dans la direction $(v, \beta)$. Notons que (ir) et (eb) portent sur l'évolution de la structure avec le temps alors que (st) ne porte que sur son état à un instant donné. L'item (st) est simplement une écriture variationnelle de l'équilibre et du critère-d'endommagement $(\phi(\varepsilon, \alpha) \leq 0)$. En effet, du fait que $\mathcal{C}_{t}$ est affine, l'écriture variationnelle de l'équilibre devient $\int_{\Omega} \sigma_{t} \cdot \varepsilon\left(v-u_{t}\right) d x=W_{t}^{e}\left(v-u_{t}\right)$ pour tout $v \in \mathcal{C}_{t}$, alors que celle du critère d'endommagement est $\int_{\Omega} \phi\left(\varepsilon\left(u_{t}\right), \alpha_{t}\right)\left(\beta-\alpha_{t}\right) d x \geq 0$ pour tout $\beta \in \mathcal{D}\left(\alpha_{t}\right)$. En additionnant et en tenant compte de (7)-(9) on obtient (st). L'item (eb) traduit la condition de cohérence (9-3). 
En pratique c'est toujours le problème incrémental, i.e. celui obtenu à partir du problème d'évolution après discrétisation en temps, que l'on résout. Pour le construire, il suffit de remplacer la variable de temps "continu" $t$ par une suite (discrète) de temps $i \in \mathbb{N}$. Le problème incrémental consiste alors à trouver, pour $i \in \mathbb{N}_{*},\left(u_{i}, \alpha_{i}\right) \in \mathcal{C}_{i} \times \mathcal{D}\left(\alpha_{i-1}\right)$ tel que

$$
\mathcal{P}_{i}^{\prime}\left(u_{i}, \alpha_{i}\right)\left(v-u_{i}, \beta-\alpha_{i}\right) \geq 0, \quad \forall(v, \beta) \in \mathcal{C}_{i} \times \mathcal{D}\left(\alpha_{i-1}\right)
$$

Le problème incrémental permet d'illustrer une propriété fondamentale des matériaux durcissants qui tient au caractère convexe de l'énergie dans ce cas-là, cf Proposition 2.3.

Proposition 3.2 Si la loi d'endommagement est une loi standard avec durcissement au sens de la Définition 2.2, alors, à chaque pas de temps où une solution existe, $\left(u_{i}, \alpha_{i}\right)$ minimise l'énergie totale $\mathcal{P}_{i}$ de la structure $\operatorname{sur} \mathcal{C}_{i} \times \mathcal{D}\left(\alpha_{i-1}\right)$.

Autrement dit, pour des matériaux durcissants le problème incrémental se ramène à une suite de problèmes de minimisation de l'énergie. Moyennant une condition d'écrouissage un peu plus forte (croissance assez rapide vers l'infini des contraintes à la limite d'élasticité quand $\alpha$ tend vers 1 ), on peut même démontrer que le problème incrémental possède une solution unique, ce qui rend les modèles d'endommagement durcissants comparables mathématiquement aux matériaux élasto-plastiques écrouissables. Cependant, en pratique, on rencontre le plus souvent des matériaux adoucissants. Dans ce cas, il est facile d'exhiber des exemples où le problème d'évolution admet soit aucune solution (propriété due à la notion de charges limites associée à la bornitude des contraintes), soit au contraire une infinité de solutions (due au caractère non monotone de la relation contrainte-déformation quand l'endommagement évolue). De plus, l'écriture des items (ir) et (eb) n'a de sens que dans la mesure où l'évolution en temps est régulière, ce qui n'est pas toujours vrai même si les données sont régulières du fait de la présence de snap-back dans la réponse globale. Comme ces pathologies subsistent même si l'on régularise les modèles en introduisant des termes non locaux, cf [2], il est nécessaire d'une part d'introduire un critère de sélection de solution basé sur le concept d'état stable et d'autre part d'avoir une formulation capable de gérer des évolutions irrégulières en temps. Cela va nous demander de changer les trois items.

3.2. La notion d'état stable. Pour des systèmes conservatifs, on peut définir les états stables du système comme ceux qui sont des minima locaux de l'énergie du système. Cette notion peut être élargie aux systèmes dissipatifs régis par des lois standards [8]. On peut introduire des conditions de stabilité plus ou moins fortes en jouant sur les choix de la distance entre états et de la taille du voisinage. On retiendra ici la notion de stabilité directionnelle qui n'utilise que la topologie de $\mathbb{R}$.

Définition 3.3 (Stabilité directionnelle) À un instant $t$ donné, un état $(u, \alpha)$ de la structure endommageable est dit stable s'il est admissible et si dans toutes les directions accessibles il existe un voisinage où tout autre état a une énergie supérieure ou égale. Autrement dit, on demande que $(u, \alpha) \in \mathcal{C}_{t} \times \mathcal{D}_{0}$ soit tel que

$$
\forall(v, \beta) \in \mathcal{C}_{t} \times \mathcal{D}(\alpha), \quad \exists \bar{h}>0, \quad \forall h \in[0, \bar{h}], \quad \mathcal{P}_{t}(u+h(v-u), \alpha+h(\beta-\alpha)) \geq \mathcal{P}_{t}(u, \alpha) .
$$

Remarquons que si l'état $\left(u_{t}, \alpha_{t}\right)$ est stable, alors en divisant l'inégalité de (ST) par $h>0$ et en passant à la limite quand $h \rightarrow 0$, on retrouve (st). Autrement dit, l'équilibre et le critère d'endommagement peuvent être vus comme des conditions nécessaires de stabilité, conditions du premier ordre en $h$. Mais elles ne sont pas en général suffisantes. Elles le sont dans le cas de matériaux durcissants, mais pas dans le cas de matériaux adoucissants. Il faut en particulier considérer aussi des conditions du second ordre en $h$, ce qui fait apparaître la notion de quotient de Rayleigh [2]. Cette étude générale fera l'objet de prochaines publications.

3.3. Le bilan d'énergie. La condition (eb) peut être vue comme une conservation de l'énergie totale quand l'endommagement évolue, le taux de restitution d'énergie élastique étant égal à la puissance dissipée. Sous cette forme, elle suppose implicitement que l'évolution de l'endommagement est régulière en temps. L'idée est d'étendre ce principe de conservation de l'énergie aux cas d'évolutions non régulières (qui sont à envisager dans les modèles adoucissants). C'est évidemment une hypothèse physique forte, car on pourrait aussi considérer que les évolutions non régulières en temps sont du ressort de la dynamique et que l'énergie cinétique doit jouer un rôle. Nous optons néanmoins pour ce choix et continuons à raisonner dans le cadre quasi-statique. L'écriture étendue de la conservation de l'énergie s'appuie sur la 
Proposition 3.4 (Bilan d'énergie) Dans une évolution régulière, l'évolution de l'énergie totale satisfait le bilan suivant

$$
\mathcal{P}_{t}\left(u_{t}, \alpha_{t}\right)=\mathcal{P}_{0}\left(u_{0}, \alpha_{0}\right)+\int_{0}^{t}\left(\int_{\Omega} \sigma_{t^{\prime}} \cdot \varepsilon\left(\dot{U}_{t^{\prime}}\right) d x-W_{t^{\prime}}^{e}\left(\dot{U}_{t^{\prime}}\right)-\dot{W}_{t^{\prime}}^{e}\left(u_{t^{\prime}}\right)\right) d t^{\prime}
$$

où $\dot{U}_{t^{\prime}}$ et $\dot{W}_{t^{\prime}}^{e}$ sont les vitesses de chargement à l'instant $t^{\prime}$.

Preuve Formelle. En dérivant par rapport à $t$ l'énergie totale, en tenant compte de (eb) et en utilisant l'équilibre, il vient

$$
\frac{d}{d t} \mathcal{P}_{t}\left(u_{t}, \alpha_{t}\right)=\int_{\Omega} \sigma_{t} \cdot \varepsilon\left(\dot{u}_{t}\right) d x-W_{t}^{e}\left(\dot{u}_{t}\right)-\dot{W}^{e}{ }_{t}\left(u_{t}\right)=\int_{\Omega} \sigma_{t} \cdot \varepsilon\left(\dot{U}_{t}\right) d x-W_{t}^{e}\left(\dot{U}_{t}\right)-\dot{W}_{t}^{e}\left(u_{t}\right) .
$$

En intégrant par rapport à $t$ on obtient (EB). (Toute la difficulté d'une "vraie" démonstration réside dans les questions de régularité en temps.)

Notons que (st) et (eb) équivalent à (st) et (EB) dans une évolution régulière, mais l'avantage de (EB) est qu'elle conserve un sens même si l'évolution envisagée n'est pas régulière car elle ne fait appel qu'à la régularité (en temps) des données $U_{t}$ et $W_{t}^{e}$. En l'adoptant on exige donc que l'énergie totale soit une fonction absolument continue de $t$ même si $t \mapsto\left(u_{t}, \alpha_{t}\right)$ est discontinue; c'est l'extension annoncée du principe de conservation de l'énergie.

3.4. La formulation étendue. On propose finalement de remplacer l'item (st) par la condition de stabilité (ST) et l'item (eb) par sa version étendue (EB). Le nouveau problème d'évolution s'écrit donc : Trouver pour tout $t \geq 0,\left(u_{t}, \alpha_{t}\right) \in \mathcal{C}_{t} \times \mathcal{D}_{0}$ tel que

$$
\left\{\begin{array}{l}
(\mathrm{IR}): t \mapsto \alpha_{t} \text { soit croissant depuis } \alpha_{0} \\
(\mathrm{ST}):\left(u_{t}, \alpha_{t}\right) \text { soit stable au sens de la Définition } 3.3 \\
(\mathrm{~EB}): \text { le bilan d'énergie soit satisfait à chaque instant. }
\end{array}\right.
$$

Il est d'un côté plus exigeant que le problème initial puisqu'on n'autorise que des états stables et d'un autre plus tolérant puisqu'on autorise des évolutions discontinues. Insistons à ce propos sur la distinction entre stabilité et non régularité. La stabilité telle qu'elle est définie ici est une propriété d'un état alors que la régularité est une propriété de l'évolution. On peut trouver des évolutions continues satisfaisant (st) mais pas (ST), de même qu'on peut trouver des évolutions discontinues qui satisfont (ST) à chaque instant.

Exemple 3.1. Pour illustrer la différence entre les deux formulations, considérons une barre à déplacement contrôlé aux extrémités. Dans le cas d'un matériau adoucissant, le problème d'évolution basé sur (ir), (st) et (eb) admet une infinité de solutions. La condition (ST) dit que toutes les solutions au delà de la phase élastique sont instables et donc sont à rejeter.

\section{Conclusion et perspectives}

Nous proposons une nouvelle formulation variationnelle du problème d'évolution de l'endommagement dans une structure faite de matériaux élastiques-fragiles standards. Le caractère standard du modèle, justifiable par le postulat de Drucker-Ilyushin, permet de tout écrire en termes de l'énergie totale, différence entre le travail de déformation (qui est alors une fonction d'état) et le travail des efforts extérieurs donnés. Elle consiste en une condition d'irréversibilité, un critère de stabilité et un bilan d'énergie. Même si elle n'a été introduite ici que pour des modèles locaux (non régularisés), son écriture se généralise facilement à des lois d'endommagement non locales. Ce sera l'objet de la deuxième partie de cette étude. 


\section{Références}

[1] H. Amor, J.-J. Marigo, and C. Maurini. Variational approach to brittle fracture with unilateral contact : numerical experiments. J. Mech. Phys. Solids, $57: 1209-1229,2009$.

[2] A. Benallal and J.-J. Marigo. Bifurcation and stability issues in gradient theories with softening. Modelling Simul. Mater. Sci. Eng., 15 :283-295, 2007.

[3] B. Bourdin, G. Francfort, and J.-J. Marigo. The variational approach to fracture. J. Elasticity, 91 :5-148, 2008.

[4] E. Lorentz and S. Andrieux. Analysis of non-local models through energetic formulations. Int. J. Solids Struct., 40 :29052936, 2003

[5] J.-J. Marigo. Formulation de l'endommagement d'un matériau élastique. C. R. Acad. Sci. Paris Sér. II, 292 (19) :13091312, 1981.

[6] J.-J. Marigo. Constitutive relations in Plasticity, Damage and Fracture Mechanics based on a Work Property. Nuclear Engineering and Design, 114 :249-272, 1989.

[7] A. Mielke. Evolution of rate-independent systems. In Evolutionary equations, volume II of Handb. Differ. Equ., pages 461-559. Elsevier/North-Holland, Amsterdam, 2005.

[8] Q. S. Nguyen. Stability and Nonlinear Solid Mechanics. Wiley \& Son, London, 2000. 